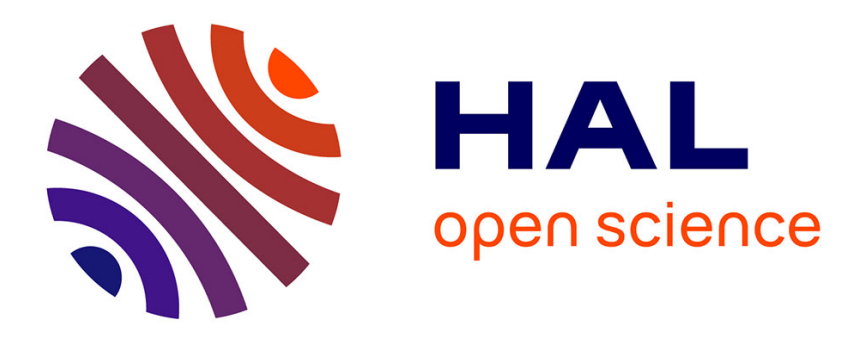

\title{
Voyageurs immobiles: la géographie idéelle des Kulung Rai du Népal
}

Grégoire Schlemmer

\section{To cite this version:}

Grégoire Schlemmer. Voyageurs immobiles : la géographie idéelle des Kulung Rai du Népal. Collection Purușārtha, 2017, pp.227-247. halshs-01487826

\section{HAL Id: halshs-01487826 \\ https://shs.hal.science/halshs-01487826}

Submitted on 13 Mar 2017

HAL is a multi-disciplinary open access archive for the deposit and dissemination of scientific research documents, whether they are published or not. The documents may come from teaching and research institutions in France or abroad, or from public or private research centers.
L'archive ouverte pluridisciplinaire HAL, est destinée au dépôt et à la diffusion de documents scientifiques de niveau recherche, publiés ou non, émanant des établissements d'enseignement et de recherche français ou étrangers, des laboratoires publics ou privés. 


\section{Voyageurs immobiles}

\section{La géographie idéelle des Kulung Rai du Népal}

Les Kulung Rai forment une société fortement territorialisée. Environ la moitié des $30000 \mathrm{Kulung}$ occupent leur vallée de référence - la vallée de l'Hongu, au pied de l'Everest -, où ils se répartissent en une dizaine de villages vivant d'agriculture en terrasse et d'un peu d'élevage, et ce depuis une vingtaine de générations, soit environ quatre d̀ cinq siècles, si l'on se fonde sur leurs généalogies. À l'image des autres groupes Rai et Kirant (les ensembles ethniques auxquels ils se rattachent), les Kulung se distinguent des autres populations dites « tribales " du centre et de l'ouest du Népal par une histoire singulière. Après leur intégration par conquête dans le royaume du Népal, à la fin du XVII's siècle, ils se sont vus reconnaître une certaine autonomie politicofoncière sur leur ancien territoire de chasse et d'essartage, wallkha-dibuka, devenu dans le langage administratif népalais kipat ${ }^{1}$. Bien que progressivement réduite, jusqu'à sa suppression vers les années 1950, cette autonomie contribue à expliquer - ou à renforcer - le lien existant entre les Kulung et leur territoire ancestral, capkua : « le lieu des mânes 》. La manière dont ils se désignent vis-à-vis des étrangers témoigne d'ailleurs de cet ancrage territorial: jusqu'aux années 1960 , seuls les chefs fonciers, tälukdār, étaient nommés Rai, tous les autres habitants s'appelant Jimi, une déformation de jamin, le « sol » et jamindär, « propriétaire foncier » (Schlemmer 2010a). Le terme même " kulung » a, en plus de son acception généalogique (les descendants des deux frères fondateurs Chemsi et Tamsi), un sens géographique, désignant alors l'ensemble des Rai de la vallée (soit les Kulung proprement dits et le groupe voisin des Nachering). Les Kulung aiment aussi à se nommer caricha, «les enfants du territoire ", un terme qui exprime un sentiment d'autochtonie. La vallée de l'Hongu reste aussi le territoire de référence pour les migrants kulung, qui l'ont quitté parfois depuis plusieurs générations. Cet ancrage territorial structure enfin le domaine religieux qui nous intéresse ici. Dans 
cette société qui ne conçoit pas les puissances spirituelles comme structurées en panthéon, le critère peut-être le plus pertinent parmi ceux qui organisent plus ou moins le monde des esprits est celui de leur origine spatiale. De manière plus générale, la zone d'habitat kulung est constitutive de leurs pratiques religieuses : celles-ci se pratiquent difficilement hors de la vallée; leurs formes épousent celles de la communauté et de son habitat.

En même temps, les Kulung aiment à se dire « d'ailleurs » et à se définir par le mouvement: «Nous étions des chasseurs, nous n'avions pas de maison, on dormait là où la nuit nous surprenait. "Ce type de propos est récurrent lorsque les Kulung parlent de leur passé. Leurs récits mentionnent également le fait que les deux frères fondateurs de la communauté occupèrent l'actuel territoire kulung en en chassant une ancienne population quil l'habitait, prônant l'acte de conquête, qui contraste avec le sentiment d'autochtonie évoqué. Apparaît ainsi, chez les Kulung, un rapport ambivalent à l'ici et à l'ailleurs. Dans le domaine religieux, cette importance de l'extériorité et du mouvement, doublée d'un attachement au territoire, est particulièrement prégnante dans les pratiques rituelles des natabom. Je ne connais pas l'étymologie de ce terme, mais il est toujours présenté par les Kulung comme désignant les étapes, les pauses ou les campements des chasseurs; je les nommerai voyages rituels ou, en raccourci, voyages 2 . Ce sont néanmoins des voyages immobiles, des parcours verbaux. Décrivons brièvement un de ces rites:

Kanchi est malade. Elle a fait venir un devin (mop), et sa divination révele que son mal est causé par l'esprit Muruos et qu'll faut un rituel complet (bim). Quelques jours plus tard, le devin revient pour accomplir le rituel. Il a lieu dans la maison, devant un autel composé d'aliments (à différentes occasions, le devin jettera sur le dos de Kanchi des poignées de graines) et de récipients. S'll s'étalt agi d'un rituel abrégé (cim), il auratt eu lieu dans la cour, et les éléments rituels auraient été réduits au minimum: un bout de bambou faisant office de gobelet et une branche d'artémise auraient suff. L'officiant s'assied derrière Kanchi, qui reste assise passivement, tournée vers une porte. Il commence ses incantations : «la fille du clan X tombant malade, ayant de la toux et le foie qui enfle, vérffiant par la divination, Muruos l'ayant rendu malade, en ce jour de la saison montante, moi devin, ayant été appelé par les sacrffants, tous les eléments nécessaires qui te sont destinés, les calebasses de bière, le van et la chope remplie de blère, la viande de vache et de gibier, étant posés, te rendant culte, je te ramène d ton lieu, au lieu de naissance des hommes; te ramenant par le chemin du vent, les âmes du corps, des épaules, du visage, des anciens et du sacrifiant, etce,je les pose dans le foyer; deputs le foyer, t'amenant sur le chemin des esprits, le chemin des mauvaises choses, t'amenant dans ton lieu par le chemin des origines, depuis la place des femmes à la porte centrale, toi, esprit, t'amenant d la fontaine, etc. S'ensuit une longue série de lieux qui sont ainsi juste mentionnés avec un verbe de mouvement. Arrivé à destination, le devin dit: t'amenant avec les mauvaises choses, regarde et prends le sang du poulet (qui est tué par secousses répétées; ll est ensuite passé au feu) 
regarde, toi, Muruos, tol quil donnes toutes ces maladies, moi, devin, dे l'endroit de te jeter dans le lac, moi devin du vent, ayant fait une double barrière, ayant fermé toutes les âmes, fermant le chemin, je reviens avec toutes les âmes, retournant d... (ll clte tous les lieux en sens inverse, jusqu'd la porte), ramenant tout d l'intérieur, la place des femmes, en notre lieu, les âmes du sacrifiant, sur le foyer, nous sommes arrivés, maintenant, dans la maison, restons / Maintenant, des hommes et des femmes sacrifiant, d la Source, toutes les forces vitales, levons / Les graines, l'argent, les buffles, le chemin, le corps, la belle voix [...], je les transforme en bien 》.

Les incantations achevées (elles auront duré 20 mn environ ${ }^{3}$ ), le poulet est cuisiné et mangé en famille, avec l'officiant, qui rentrera ensuite chez lui, tandis que le malade espérera sa guérison.

Les incantations qui forment ces rituels narrent un voyage, composé essentiellement de noms d'étapes (leur nombre varie selon le rite et, pour une part, selon l'officiant qui l'exécute, mais s'étendent de dix à quarante noms 4); il s'agit d'aller chercher des bienfaits ou de rejeter des mauvais sorts, de prendre (du bien) ou d'éloigner quelque chose (du mauvais). Nous allons voir que la plupart des étapes de ces voyages rituels sont (plus ou moins) localisables, et que l'on pourrait suivre ces trajets sur une carte (même si tous les noms de lieux ne sont pas toujours bien identifiés et que, au fur et à mesure que l'on avance le long du parcours, ils renvoient de plus en plus à des lieux uniquement mythiques).

Tableau. Les différents voyages

(tableau et schémas de Grégoire Schlemmer)

\begin{tabular}{|c|c|c|c|}
\hline Rituels impliquant un voyage & $\begin{array}{c}\text { Nombre d'étapes } \\
\text { (approx.) }\end{array}$ & Destination & Déplacement \\
\hline Năgi et Dedam & 40 & & Souterrain \\
\hline Jikham (appeler la chance) & 20 & & \\
\hline Séance divinatoire (selemop, demop) & 20 & Yowna, la Source & Aérien \\
\hline Diburim (esprit de la chasse) & $?$ & & \\
\hline Serbim (maladie) & $?$ & (ou Salpa/Kidili?) & Terrestre \\
\hline Sapphom (rejet des infortunes) & 10 & & \\
\hline Srap (malédiction) & 10 & Sobolu, l'Embouchure & \\
\hline Funérailles & 35 & & Aérien \\
\hline Mauvais morts & $?$ & & Terrestre \\
\hline Calim (rite agraire) & 35 & & \\
\hline Muruos (esprit-maladie) & 35 & & \\
\hline Lalao (esprit maitre de la forêt) & 35 & Minapongkho, l'origine & \\
\hline Dunima (esprit-maladie) & 15 & Dokoma-Danda & Terrestre \\
\hline Tome (esprit des mortes en couches) & 35 & & \\
\hline Sampkowademong (esprit-maladie) & 45 & Hatya-Golhe & \\
\hline Kalko-mumang (esprit-maladie) & & Matryd-Goine & \\
\hline
\end{tabular}


Ces incantations sont prononcées par un officiant; le bénéficiaire du rituel - un individu ou toute une famille - demeurant passif. Il ne s'agit pas d'un voyage de l'âme ; même si tout laisse à penser que « quelque chose » de l'officiant s'en va, ce n'est pas confirmé par les officiants; ces voyages se font « par le discours (ridum) n. Ceci n'empêche pas que ces rites sont perçus comme dotés d'efficacité, et sont même source de danger (ce pourquoi les âmes doivent être mises en sécurité sur le foyer et l'officiant doit, en jetant des graines de sarrasin par-dessus ses épaules, se protéger des flèches que lui enverraient des adversaires en des lieux précis des voyages). Cette efficacité du rite, qui le rend potentiellement dangereux, provient du fait que ce voyage est prononcé, non pas dans la langue kulung ordinaire, mais en sumning, la langue (ning) des origines (sum). Composée de mots doublés, d'images et de termes archaiques, cette langue est en fait un parler rituel, compréhensible pour qui tend l'oreille. Les noms d'étapes sont ainsi ce que l'on appelle des dapsing, ces noms ancestraux, à l'efficacité proche des formules magiques mantra qui activent le pouvoir inhérent aux choses.

Ces voyages révèlent la manière dont les Kulung découpent et conçoivent leur environnement géographique, organisent l'espace et les lieux qu'ils retiennent et valorisent (soit, plus que les différentes étapes parcourues, les destinations de ces voyages et leur direction), autant de données qui s'articulent à un certain rapport à l'extériorité et à l'altérité. On présentera les espaces parcourus, ce qu'en disent les Kulung et ce qu'ils y font, afin de dégager ce qu'évoquent ces voyages et ce qui y est associé. De la maison à leurs différentes destinations (le village et la vallée, l'amont, l'aval-embouchure, l'aval-origine, le chemin des esprits étrangers), on étudiera les évocations et associations ressortant des principales étapes et destinations de ces voyages.

\section{L'espace habité}

Énoncés soit dans la cour de la maison, soit dans l'unique pièce de la maison, le lieu du sacrifiant par excellence (kuo morugo), les voyages commencent toujours par la demeure du sacrifiant. Le dénuement de l'intérieur des maisons, au mobilier très limité, masque la complexité de leur organisation interne. Clarifier le fonctionnement de cet espace structuré, hiérarchisé et empli de règles, d'obligations et d'interdits, permet de mettre au jour quelques-unes des coordonnées et principes structurants de la géographie rituelle kulung 5 .

Seul le rez-de-chaussée de la maison est habité. Interdit d'accès à tout étranger à la maisonnée, l'étage sert d'entrepôt à grains (soit les richesses de la maisonnée) et aux esprits de la maison (qui aident à la prospérité). Sans aucune cloison interne, le rez-de-chaussée n'en est pas moins divisé en 
plusieurs espaces, selon deux lignes invisibles. La première marque la division entre le haut et le bas, selon un critère topographique qui sied aux pays montagneux : toutes les maisons sont construites parallèlement à la pente, et donc à la rivière, et l'on considère que la partie située vers la crête est « haute " (tokka), celle du côté de la rivière, « basse » (tukka). La seconde marque une division entre la partie avant/arrière ou aval/amont ${ }^{6}$. Ces critères créent ainsi une division de l'espace interne de la maison en quatre quarts marquant trois principaux espaces (haut/avant, mosum; haut/arrière, mamatusko; «le chemin du bas ", lamcinipu - la division avant et arrière du bas étant faiblement marquée). Le centre de cette partition de l'espace est marqué par un pilier (qui peut être absent mais qui doit au moins être placé temporairement lors de l'inauguration de la maison) et l'un des coins du foyer (daplo).

\section{L'espace masculin}

Elément le plus important de la maison, le foyer occupe la partie haut/avant. Point de départ de tout voyage rituel (une sorte de kilomètre zéro), il condense en lui la division tripartite de l'espace de la maisonnée. Il s'agit en effet d'une fosse carrée creusée dans le sol et bordée de pierres à l'intérieur de laquelle trois pierres sont disposées en forme de triangle isocèle, renvoyant chacune à l'espace qui lui est opposé: la " pierre père " (pakholung), espace masculin, au haut/avant; la « pierre mère " (makholung), espace féminin, au haut/arrière; la "pierre-racine fortement ancrée " (sambelung-bulolung), espace autre (des alliés, des visiteurs), qui forme la partie basse de la maison. Un récit mythique de la fondation du foyer associe chacune de ces pierres à un élément de parenté: le maître de maison (représentant la patrifiliation), le beau-père (soit le donneur de femmes, ici le Nāgi, voir infra) et les gendres (soit les preneurs de femmes), comme si le foyer et ses trois pierres, autonomes mais inséparables si l'on veut y faire bouillir la marmite qui assure la survie de la famille et la reproduction de la lignée, marquaient la nécessaire collaboration des groupes. Ceci, associé aux propriétés de la pierre - marqueur de solidité, durée et continuité - et du feu - qui produit chaleur, lumière et cuisson - fait que le foyer est doté d'une grande sacralité. Pour reprendre les propos d'un ancien, «le foyer, c'est notre vrale divinité, la plus grande des Kulung. Ce feu, c'est comme le divin. Partout où les hommes vont, ils s'installent en un lieu, et posent ces trois pierres pour le feu et le repas. C'est pourquoi nous devons rendre culte au foyer ". L'ancien fait ici référence aux libations de bière effectuées sur les pierres du foyer lors d'événements auspicieux (inauguration d'une nouvelle maison, mariage, rite au Nägi) en demandant à la pierre-père de protéger et d'augmenter la force vitale, à la pierre-mère d'apporter les bienfaits et à la pierre-racine d'éloigner les infortunes. Ces libations s'opèrent dans le sens des aiguilles d'une montre, tout comme la spirale que dessine le voyage à l'intérieur de la maison. Lors des voyages aussi, le 
foyer joue un rôle protecteur puisque, avant la plupart des voyages, l'officiant annonce dans ses incantations rituelles qu'il dépose les âmes sur le foyer. Il énumère alors une longue liste d'âmes: les siennes, celles des participants et de tous leurs parents, des animaux domestiques, et de tout ce qui a une âme. Il va en effet aller sur le chemin dangereux des esprits, et leur demande de ne pas le suivre. Les âmes en sécurité sur le foyer, alors commence le voyage.

La première étape du voyage, c'est le mosum, que je traduis par «place haute . Cet espace, situé entre le foyer et le mur qui donne vers la crête (partie haut/avant), est réservé au maître de maison et aux anciens. Il est interdit à toute femme nubile, en raison de l'incompatibilité entre le sang du gibier mort que l'on devait déposer sur ce lieu, et celui, procréateur, des femmes. Le gibier nourrissait les vivants et servait d'offrande aux ancêtres. Ainsi, lors des libations aux trois pierres du foyer, une patte de daim est posée sur une petite étagère, dampe, qu'on appellera désormais « autel domestique ». Simple planchette de bois située sur le mur, un peu en amont du foyer, entre les espaces masculin et féminin, elle fait office d'autel aux ancêtres. Lors des rituels de funérailles, on dit " poser " l'esprit du mort sur l'étagère qui se situe dans la maison qu'il habitait. Comme les trois pierres font le foyer, ces trois lieux - foyer, place haute et étagère - sont inséparables et renvoient à la figure du maître de maison, chasseur, sous les auspices de ses ancêtres.

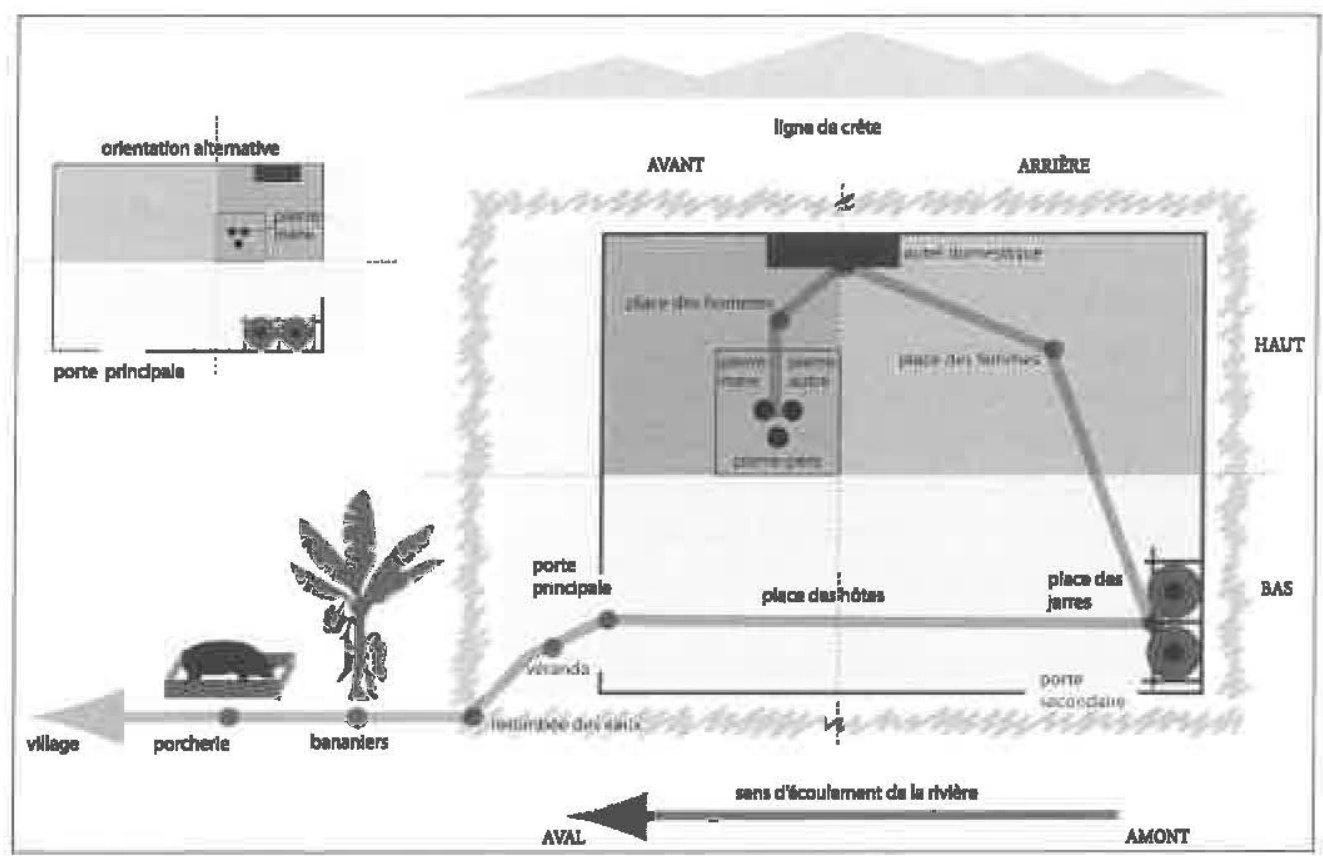

Schema 1. Organisation de l'espace domestique 


\section{De l'espace féminin au dehors}

La seconde étape est le coin haut/arrière, soit l'espace féminin, la place réservée à l'épouse, complément indispensable à l'homme pour fonder la maison. À elle est associée la part procréatrice et nourricière. C'est de cette place que l'épouse sert les repas aux membres de la maison et aux hôtes.

Cette place se trouve aussi à proximité des jarres d'eau et de bière, utilisées pour les libations au foyer effectuées lors du très important rituel domestique au Nāgi ${ }^{7}$. Comme la place haute et l'autel domestique, le lieu des jarres est associé aux ancêtres et à la prospérité. Ils ne forment des étapes que pour le voyage rituel au Nāgi; on dit que les autres esprits, néfastes, les détruiraient. Lors des autres rites, on passe donc du foyer à l'espace féminin, puis à la porte. La partie basse où se trouvent les jarres représente déjà un peu le dehors. Il s'agit d'un espace de passage, et c'est là que les personnes extérieures à la maisonnée sont invitées à s'asseoir (du bas-arrière au bas-avant selon le degré d'importance croissant attribué à l'hôte).

La porte principale marque la fin de cet espace ordonné, mais non encore véritablement le dehors. Il reste à l'officiant à franchir, donc nommer, la véranda et la ligne de retombée des eaux qui entoure la maison et marque les limites spatiales du bâtiment. Cette dernière délimite elle aussi une zone de transition; ce n'est ni le dedans, ni le dehors. C'est peut-être pourquoi les mort-nés - qui ne sont pas vraiment morts, car ils ne furent pas vraiment vivants - y sont enterrés dessous, sans parole ni cérémoniel. Au-delà, se trouve la cour ouverte, qui s'étend devant la maison. Malgré son usage quotidien, elle ne constitue pas une étape des voyages, à l'inverse de l'enclos pour les porcs nécessaires au sacrifice et, avant elle, du bosquet de bananiers dont les feuilles agitées aux vents font fuir les maladies.

On retiendra que la maison semble organisée selon un principe tripartite opérant à plusieurs niveaux : les trois pierres forment le foyer lequel, avec la place des hommes et l'autel domestique, forme la partie haut/avant; ellemême, combinée avec la partie des femmes, et la partie basse forme l'intérieur de la maison. Entre chacun de ces éléments, une transition (le bord du foyer, l'autel domestique, la place des jarres), implique qu'un élément d'extériorité est inclus dans ce qui définit le dedans. De surcroît, le plan domestique forme une sorte de matrice du champ de représentation spatiale des Kulung. Les coordonnées et les valeurs qui structurent l'espace de la maisonnée (haut et bas, amont et aval, importance des consanguins et des alliés, recherche de la prospérité et éloignement des dangers, etc.) se retrouvent dans la géographile des voyages rituels qui s'étendent hors de la maison et que nous allons décrire maintenant. 


\section{Le village et la vallée}

Il n'y a pas de transition brutale entre un dedans et un dehors; du foyer à la porcherie, l'extériorité se marque par paliers, par étapes - celles du voyage. Après la maison, viennent une ou deux étapes relatives à l'espace public, qui est l'espace du village. Lors des voyages, l'étape qui correspond au village, tel, n'est pourtant pas nommée ainsi, mais par le terme car (qui peut être utilisé en doublé avec tel: carcha-telpucha désignera ainsi les villageois « enfants du territoire, enfants du village »), ou par le terme de chemin, lam, ou chelamdelam en langue des origines. Pour reprendre l'explication d'un ancien: "Chelam, c'est les chemins, là où passent les chiens, qui circulent entre les maisons; delam, c'est là où passent les chats, qui circulent dans la maison. Chelam, c'est l'espace public, qui est sous le regard des autres, où l'on est jugé. On parle de chelam quand on se rend dans un lieu peuplé, comme le marché, où l'on va paré de ses plus beaux habits ". Le village est la somme de ces espaces privés que forment les maisons et les réseaux les connectant. La notion de car se rapproche plus de l'idée de territoire, dans toutes ses dimensions. La racine cari évoque la terre: carima, caridu: la terre, le monde (carikuwa : la fontaine potable, selon Gaenszle 1999), ainsi que, par extension, le lieu d'habitation, le village et ses champs (champs qui semblent non compris dans la notion de tel). Ce territoire peut aussi être nommé par son nom rituel: ainsi le village de Bung - où furent enregistrés la plupart des voyages rituels est Tomane caridu, « la terre [du clan] Tomachha \#. Le grand nombre de noms rituels de lieu dans la vallée fait de cette dernière un espace saturé de sens, un renvoi permanent et tangible au passé des Kulung.

Ce territoire est associé à l'identité de la personne: car an pokakeu, «ce territoire t'a fait naître " dit-on lors des naissances, tandis que lors des funérailles, on dit au mort an car luletake : « Tu as quitté ce territoire ». On dit aussi que l'on tombe souvent malade si l'on reste trop longtemps hors du territoire. Le grand rituel au Nāgi (dedam) implique d'ailleurs une divination qui a pour finalité de retrouver les âmes dans la maison en énumérant dans l'ordre les étapes domestiques du voyage. Si elles ne sont pas sur l'autel domestique, c'est le signe d'un problème. Plus elles sont loin, plus le danger est grand; passée la limite du village, la mort est proche, comme si l'on n'était en sécurité que dans ses frontières.

\section{Hors de la vallée}

Contrairement à ce que l'on a pu observer chez de nombreuses populations d'Asie, ni le monde céleste, ni le monde souterrain ne jouent un rôle très important dans le domaine religieux des Kulung. Le ciel n'est ni la destination 
des morts, ni la résidence des divinités. De même, s'il existe des forces chthoniennes (tel le Nāgi), il n'existe pas de conception relative d̀ un « monde souterrain ». En conséquence, il n'est nulle idée de paradis ou d'enfer: pour les Kulung, le monde des esprits se situe avant tout, comme celui des hommes, sur terre. Pour autant, cela n'exclut pas nécessairement l'idée d'une hiérarchisation verticale du monde: l'Himalaya est suffisamment accidenté pour favoriser une opposition entre le haut et le bas ${ }^{8}$. On peut regrouper les voyages selon leurs directions, qui ne sont pas nommées par les points cardinaux, peu mobilisés chez les Kulung, mais par le couple amont/aval (yownal yowmer): ceux qui vont vers l'amont pour aller chercher des bonnes choses, et ceux qui vont vers l'aval pour éloigner les mauvaises.

\section{L'amont (yowna), source du monde}

Lors du rite au Nāgi, des séances divinatoires des chamanes (ou devins) et du rite pour Diburim (l'esprit de la chasse), le voyage rituel prend la direction de yowna. Chacun de ces rites implique un mode de locomotion spécifique: lors de Diburim, l'officiant dity aller « en marchant " (lolanna), tandis que le devin dit se déplacer « en allant par-dessus » (cocayala) et que l'officiant au Nāgi y va " par dedans » (gocayala), comme s'il effectuait ses étapes au fond de la rivière. Même si les destinations de ces voyages varient, ils sont dits conduire vers la même direction, à Yowna. Le chemin d'accès en est donc tout naturellement la remontée de la rivière qui traverse la vallée, même si les officiants s'offrent quelques détours.

On ne connaît pas de localisation précise pour Yowna (de yow, rivière), que je traduirai par la "Source ». Plusieurs personnes ont tenté l'expédition de remonter la rivière de la vallée vers le nord, afin de voir à quoi ressemblait la Source; toutes, bien sâr, échouèrent. C'est que la Source désigne un lac source de toutes les rivières, associé aux hautes montagnes, points les plus proches du ciel. Elle est fréquemment associée aux montagnes archétypiques qui forment le toit du monde. Ce sont le Mont Méru et, surtout, le Kailash - non pas le Kailash géographique, situé sur le plateau tibétain, au nord-ouest du Népal, mais le Kailash comme montagne idéal-type. À l'idée de hauteur qu'évoquent ces terres éloignées de l'habitat humain, sont associés le silence et la pureté. Ce sont les lieux des sources et des lacs, à l'eau calme et limpide, parfois habités par des « déesses » (devi) ou autres esprits du monde sauvage (ophidiens nāg, esprits chasseurs sikärî).

Lorsqu'un voyage prend la direction de yowna, il est positivement connoté. Lors des séances divinatoires, le voyage effectué par le devin a pour objectif de récupérer une substance magique (khow) en retraçant l'itinéraire suivi, dans le mythe, par deux oiseaux émissaires partis à la recherche du premier devin. Le voyage fait lors du rite à Diburim a pour finalité d'aller chercher les 
" fleurs » ou « chance » (bung) pour la chasse. Quant au voyage jikham effectué lors du rite au Nāgi, il consiste en une énumération de noms de plantes, en partant de celles poussant dans les basses terres pour citer progressivement celles se trouvant aux sommets des montagnes. Il s'effectue en vue d'obtenir prospérité (ji) et force vitale (sai).

Yowna est ainsi un lieu considéré comme la source de la vie et de la prospérité. Par contraste, les voyages qui vont vers l'aval impliquent la résolution d'un problème préalable: ils font suite à une infortune, qu'il faut ramener à son lieu d'origine. S'enchâssent ici deux notions distinctes mais reliées: le bas et l'origine.

\section{L'aval, chemin des origines}

Il représente l'embouchure de toutes les rivières. Nommé MinapongkhoHalkhumbu (dorénavant Minaponkho), soit « lieu du soleil rouge où l'humain s'est levé ", c'est le lieu de naissance des ancêtres. C'est là que l'officiant accomplissant les rites lors des funérailles, le lachocha, va "en volant " (puperna) « montrer le chemin " (lam im) au mort (soit le chemin par lequel ses ancêtres sont venus) et dépose, " par le discours ", ses " ossements ancestraux » (minatupri), avant de le ramener ensuite dans la vallée. Le voyage de migration des ancêtres est donc retracé à rebours lors des funérailles. Il est géo-localisable, jusqu'à ne plus évoquer que des lieux mythiques (ex. Hadipongko-Barapongko: le lieu de la naissance du bétail). Minaponkho est présenté comme étant un immense lac (Diritti-Hotodi), ou un océan, situé au sud, dans la plaine indienne. C'est à cet endroit, le lac-océan, que débute l'histoire de l'humanité.

Il règne une certaine confusion concernant la localisation de Minapongko. Certains le situent à Vārāha-Kșetra, ancien temple hindou (Sircar 1960) et haut lieu de pelerinage, associé à la confluence de la Tamur, de la Sun et de l'Arun (la jonction de ces trois rivières se trouve en fait un peu en aval de ce temple). Pour d'autres, Minapongkho correspond d̀ la Saptakosi (littéralement « les sept rivières »), fleuve principalement constitué des trois rivières précédemment citées qui se forme à la jonction des montagnes himalayennes et de la plaine indo-gangétique. Une fois qu'il atteint la plaine, à quelques kilomètres de Vārāha-Kșetra, il devient alors le plus large fleuve du Népal, et l'on comprend que, pour des montagnards qui n'ont jamais vu la mer, il soit associé à l'océan. Mais pour certains autres, enfin, Minapongkho désigne Käșī, le nom religleux de Bénarès, ville dont on sait le caractère particulièrement sacré pour les hindous. Ces confusions heuristiques permettent de cumuler les valeurs dont les lieux avec lesquels s'opère la confusion sont porteurs. 
Ce cadre géographique est donc structuré par un réseau hydrographique qui donne le schéma suivant: Yowna, la Source, au nord, en haut d'une montagne, le lieu des divinités. À l'opposé, le bas, c'est le sud, la plaine, les rivières et l'océan. Pour autant, les récits mythiques semblent suggérer qu'il fut un temps où les deux lieux ne firent qu'un. Dans le mythe, à l'origine du monde, il n'est que l'eau, sous la forme d'un lac, nommé Diritti-Hotodi, dans lequel vit ou vivent le ou les Nāgi. C'est à partir de ce lac originel, ni en haut, ni en bas, ou les deux à la fois, que l'humanité sera créé. Il s'y trouvait une motte de terre dérivant sur l'eau, sur laquelle germa une touffe d'herbe dans laquelle se trouva le premier être vivant, l'insecte Hohorem, mère de la première humaine, qui enfantera l'humanité: Ninam Ridum ( «la généalogie céleste »). Le lac d'origine, associé aux Nāgi, laisse à penser qu'il s'agit de la Source. Mais c'est à Minapongkho que l'offlciant funéraire situe l'événement, comme si la motte d'herbe avait dérivé de la Source jusqu'à l'embouchure 9 ou que ce lac originel s'était distendu et polarisé, comme si la notion de lieux distincts apparaissait ultérieurement, fournissant alors l'axe temporel et spatial. Et c'est le long de cet axe que se développe de l'humanité car, par la suite, les ancêtres des Kulung vont en quelque sorte remonter l'axe

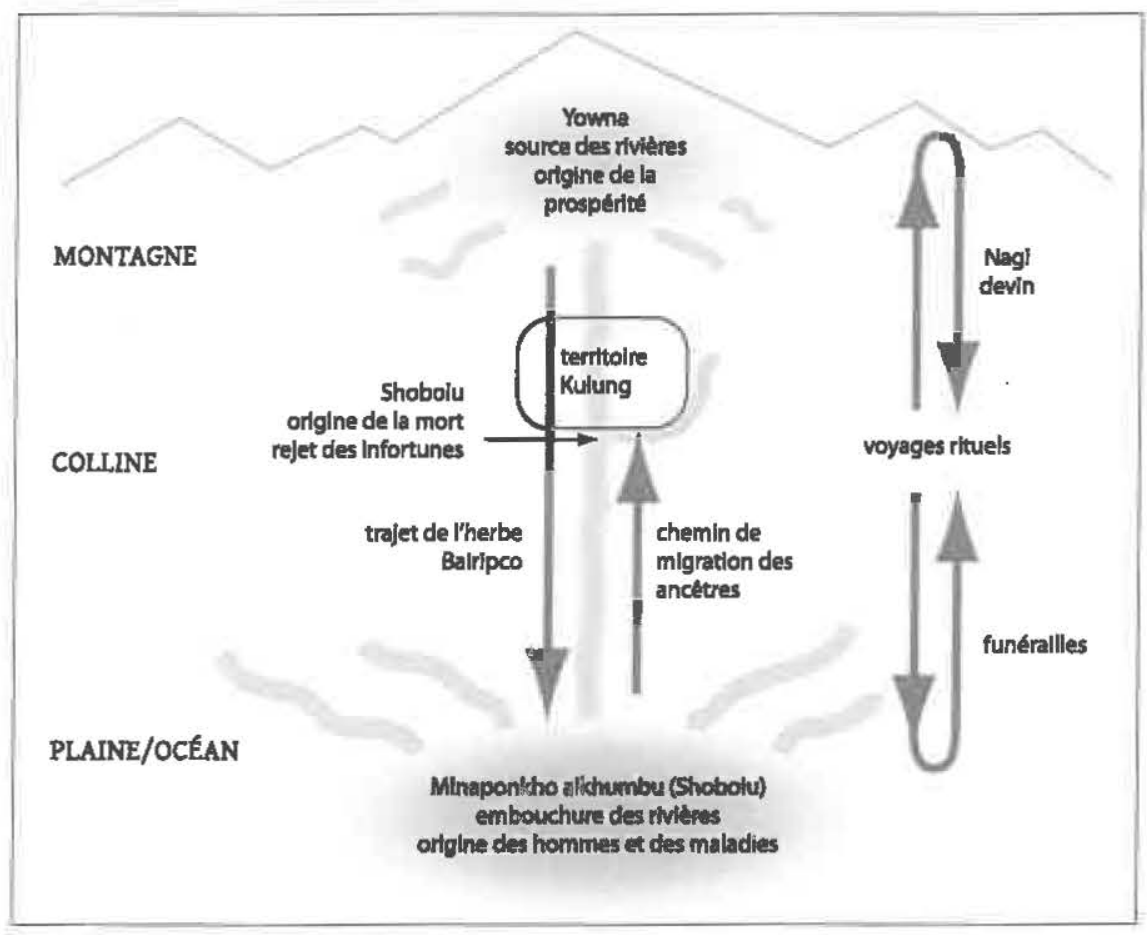

Schéma 2. Représentation schématlque de la topographie mythique 
de la rivière jusqu'à une position plus ou moins médiane entre la source et l'embouchure, pour y fonder un habitat dans la région qu'ils occupent encore actuellement. C'est du moins ce que nous narrent les mythes kulung.

\section{L'aval (yowmer), chemin de l'embouchure}

Tous les voyages qui mènent vers le bas ne renvoient pas à l'origine de l'humanité. L'aval, c'est aussi les bas-fonds, la chaleur, l'impureté et le lieu des maladies et des épidémies. Suivant les métamorphoses des cours d'eau - des eaux calmes et pures du toit du monde aux torrents boueux et déchaînés quil grondent dans les fonds escarpés des vallées - les valeurs positives associées à l'amont se transforment en valeurs négatives. Si l'embouchure du monde est le chemin que l'on montre aux morts, c'est aussi celui par lequel sont venues les premières maladies. Ce chemin est parcouru (cette fois-ci "en marchant ", lolanna), pour le rituel de l'esprit Muruos, aussi qualifié de sumnimang, l'esprit/maladie des temps premiers, comme si les maladies avaient elles aussi suivi les hommes dans leurs déplacements. On notera que, peut-être par souci de différentiation, ce lieu n'est alors pas nommé Minapongkho, mais Shobolu, qui désigne aussi la pointe extrême du sud de la vallée qui forme la limite du territoire kulung. Sorte de métonymie géographique, de "méto-topie ", de Minaponkho, c'est à cette embouchure que se rendent les officiants par un petit voyage rituel pour y évacuer infortunes et esprits néfastes et ériger verbalement, " par le discours ", une barrière pour empêcher le retour de ces influences néfastes. Cette action rituelle est nommée sapphom, littéralement « égarer intentionnellement, laisser derrière soi \%. Les rivières de bas-fonds escarpés et difficilement franchissables sont ici frontières pour les humains, chemins pour les maladies, et des sortes de dépotoirs à infortunes (qui sont, de facto, envoyées chez les voisins). Lors du voyage sapphom, la rivière joue alors le rôle de véhicule par où l'on rejette les malheurs des corps et des maisons ${ }^{10}$.

\section{Le chemin des esprits étrangers}

Le voyage comme technique de rejet d'infortunes se retrouve dans tout un ensemble d'autres rituels, adressés à des esprits, les mang, terme que, hors contexte, les Kulung traduisent par maladie, bimar - métonymie illustrant bien l'aspect négatif associé au domaine des esprits. $\AA$ la différence de ceux précédemment évoqués, ces parcours et ces esprits ne sont pas intégrés dans le mythe, et la direction que prennent les voyages n'entre pas dans l'opposition entre le haut et le bas, puisqu'il s'agit de ramener, telle une reconduction d'étrangers, ces esprits chez eux, ce "chez eux " pouvant se situer dans n'importe quelle direction. Mais, comme lors des voyages effectués en direction de l'aval, ces esprits et leurs rituels contiennent un aspect négatif, 
puisqu'il s'agit d'expulsion. «T'amenant par le chemin des esprits, allant en écartant les fourrés ", " allant sur le chemin enchevêtré de broussailles, sur le chemin des mauvaises choses ", dit parfois l'officiant. On notera aussi que deux de ces destinations sont des lieux marqués pour les Kulung : Hatya Gola (important lieu de passage des caravanes tibétaines) et les routes du sel, Dolakha (centre Newar avec lequel les Kulung semblent avoir des relations rituelles, et peut-être politiques, depuis longtemps).

La plupart des esprits singularisés qui apportent des infortunes ne sont pas des esprits de morts kulung, mais sont dits venir d'autres ensembles humains : autres clans, autres villages, autres groupes. Il en est ainsi de l'esprit Dunima, qui était une femme Khaling Rai; de Mamang, la fille d'un Rai et d'une Sherpa; de Gem, l'esprit Sotang Ral, etc. Certains de ces esprits vont jusqu'à être nommés du nom même de ces groupes: par exemple, les noms des esprits Yamphumang, Limbumang, Kalko (Khaling) et Sam sont des noms de groupes kirant auxquels est accolé le suffixe mang. Plus encore qu'associés aux morts des groupes dont ils dont ils proviendraient, ces esprits semblent être une personnification de l'altérité dangereuse intrinsèquement liée à ces populations. Souvent frustrés par des désirs insatisfaits et morts de façon violente - telles Dunima, morte pendue, ou Mamang, morte en couches, ces défunts portent un nom spécifique et sont crédités d'une histoire particulière, censée avoir été la leur, et qui leur survit. Mais ce qui compte avant tout aux yeux des Kulung, c'est que ces esprits sont source de problèmes. Ils se sont manifestés aux hommes en les soumettant à des maladies incessantes. Ils font partie - avec les mauvais morts, les esprits forestiers et les esprits de sorciers - des dangereux esprits errants du dehors, sans autel fixe. Envieux, jaloux, prédateurs, ces esprits sont alors dits prélever leur nourriture sur les vivants en leur volant des âmes, qu'il faut tenter de racheter par un sacrifice (Schlemmer 2010b).

Plusieurs de ces esprits sont des femmes issues d'autres groupes mais qui se sont mariées à des Kulung; quoi qu'il en soit, tous se transmettent, telles des maladies, par les femmes. Les femmes, forcément étrangères à la patrilignée qu'elles rejoignent par leur mariage, amènent avec elles les infortunes qu'il faudra ensuite expulser à l'aide d'un voyage. Car la femme est d'ailleurs (autre clan, autre village, autre groupe), et donc ambivalente. Elle est nécessaire à sa survie en vertu de son pouvoir reproducteur et nourricier, mais elle est source de danger.

On note que les rituels kulung établissent de nombreux parallèles entre la femme et le gibier. Comme pour ce dernier, c'est par la chair de la femme, et l'écoulement de son sang, que le groupe se reproduit. Extérleure au groupe, il faut aller la chercher (c'est ainsi que Wehilim, la fille de l'esprit le plus important des Kulung, le Nāgi, et femme du héros-ancêtre Kokcilip, fut 
attrapée à la pêche). Pour ce faire, il faut concilier ses propriétaires (beauxparents, man̂tres du gibier), qui restent néanmoins toujours potentiellement dangereux. Ceci fait que les femmes sont source de prospérité mais aussi de danger. Dolemkhu est aussi une femme, associée à la forêt, parfois à un animal, mais c'est devenu un esprit de la maison qui dispense la prospérité. Étant déjà là, elle est sans voyage rituel. Il en va de même pour Purbe, un autre esprit de la maison, mais dont les Kulung se souviennent qu'il fut un temps un esprit errant, que l'on expulsait à l'aide d'un voyage, avant qu'll se pacifie puis qu'il soit fixé dans les maisons; son voyage rituel s'est depuis perdu. Ainsi les esprits féminins et autres que l'on ramène chez eux par des voyages rituels comme on reconduirait un clandestin, peuvent être perçus comme la contrepartie négative de la nécessaire ouverture du clan sur l'extérieur, d'où vient la vie, la richesse, la prospérité.

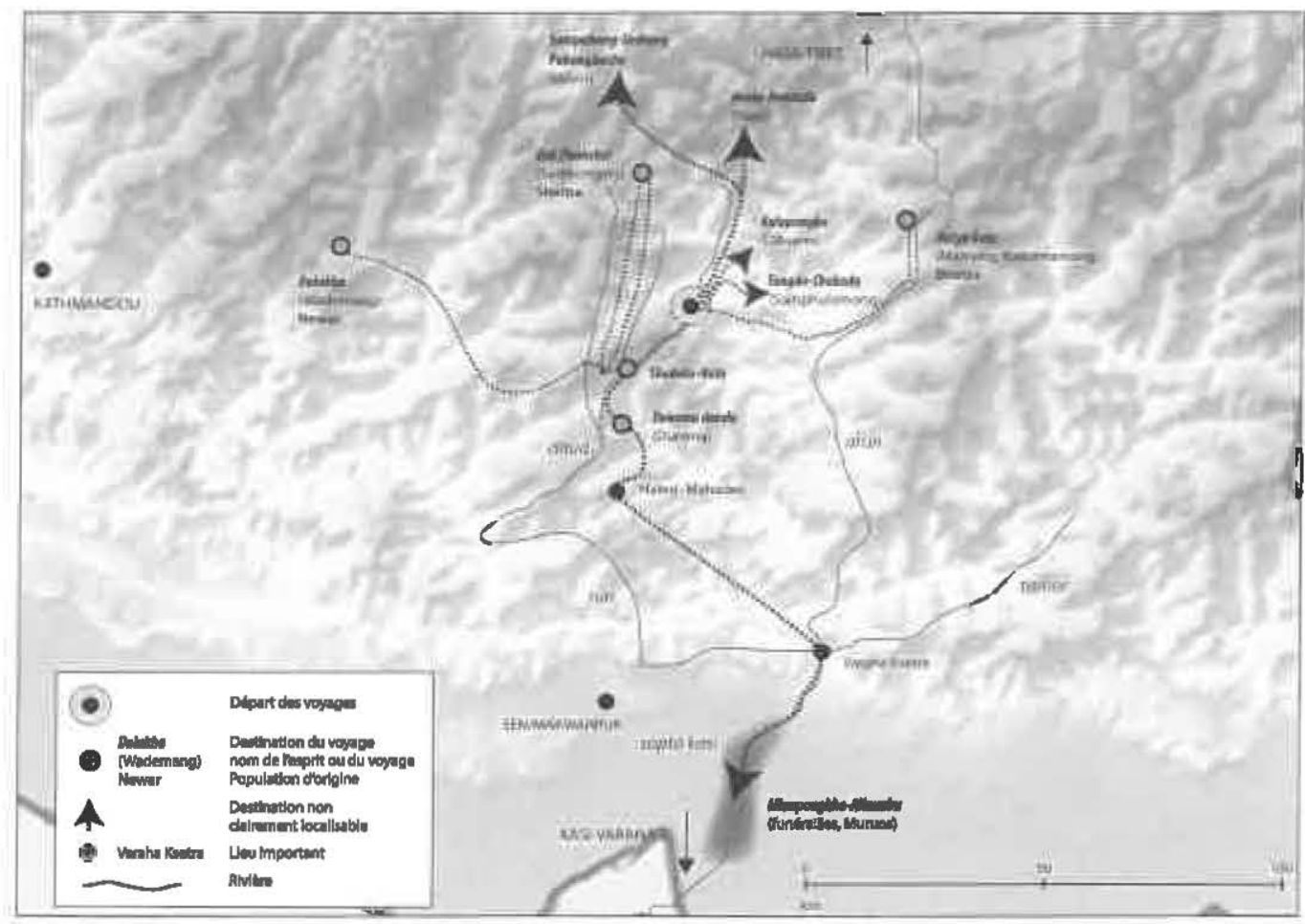

Schama 3. Cartographie des voyages rituels 


\section{Les voyages replacés dans leur contexte rituel}

Pourquoi les Kulung font-ils des natabom? Pourquoi ramener les esprits responsables d'infortunes chez eux en listant tout un ensemble d'étapes au lieu de se contenter de nommer la destination finale et de les chasser? Si ces questions restent pour moi sans réponse, on peut néanmoins, en guise de conclusion, tirer quelques enseignements de ces voyages, notamment en les comparant et contrastant à d'autres pratiques rituelles, qui les éclairent sous des angles nouveaux.

\section{Le parcours}

Ni monde céleste, ni étage souterrain, l'univers religieux kulung s'inscrit entièrement sur la terre, du pied de l'Himalaya à son sommet, sur une distance d'une centaine de kilomètres autour de la région qu'habitent encore aujourd'hui les Kulung. En grande partie localisables sur une carte, les trajectoires de ces voyages révèlent une superposition d'une géographie mythique et rituelle à la géographie physique, la topographie, combinant ainsi des notions cosmologiques avec une géographie connue. Plus encore, le voyage inscrit l'histoire dans l'espace géographique. Les chemins parcourus sont les chemins d'origine de tels esprits, «le chemin de toujours ", qui renvoie à une idée des origines, au chemin ancestral ${ }^{11}$; et plusieurs de ces voyages renvoient à un précédent mythique. Avancer dans l'espace, c'est alors avancer dans le passé, remonter dans le temps: la réalisation d'un voyage rituel est la répétition d'une action qui,jadis, a été conduite concrètement, et sa répétition reproduit l'efficacité (et les dangers) de ce référent. Sortes de voyages spatio-temporels, ils actualisent ainsi la mémoire d'événements géo-localisés. Notons aussi que plus on s'éloigne vers des terres mal connues, plus il est de correspondance entre la géographie kulung et des hauts lieux de renoms: Minapongko/Bénarès, Yowna/Kailash, Halesi, Vārāha-Kșetra. C'est aux marges de l'espace connu que se fait l'ancrage avec les grands lieux de référence des mondes indo-tibétains.

Relevons ensuite l'insistance sur le parcours, qui forme l'essence même du voyage. Les chemins que narrent les voyages ont dâ être connus, puisqu'on s'en souvient, mais les étapes ne sont ni historiquement, ni rituellement, ni spirituellement marquées. Ce sont des haltes, comme les marcheurs en font, où des repères, tels que mentionnés lors de description de chemins. C'est qu'un être semble fait par le chemin d'où il vient: pas seulement par l'origine, mais par le parcours, même si l'origine reste le plus important, l'identité de la personne se référant à son lieu. En écoutant les incantations, le malade entend son mal, lié à un esprit qui vient de loin, être chassé et s'éloigner, étape par étape, pour revenir à sa source; puis il entend l'officiant revenir avec des composantes de lui-même. 
Le voyage évoque la récitation par des officiants lors de certains rituels des lignées généalogiques électives (ridum) qui retrace par quel chemin leur esprit électeur est venu. En fait de lignée généalogique, il s'agit de la récitation de tous les noms de clans des donneurs de femmes de leur patri-lignée, de celle de leur mère et de leur femme, sur un minimum de sept générations. Ces généalogies, qui inscrivent l'individu dans ce vaste réseau formé par les alliances passées par ses ascendants, sont propres à chacun. C'est donc un chemin généalogique, dont chaque nom de clan forme une étape, qui particularise, telle une carte d'identité, chaque individu d'une patri-lignée. Comme ces récitations de lignées, les voyages associent l'individu à une étape dans un grand tout.

\section{Le territoire}

Pourquoi le village et le territoire qu'occupe le sacrifiant, qui forment des lieux saturés de sens et d'histoire, ne sont pourtant que rapidement évoqués lors des voyages? Peut-être est-ce parce que les enjeux ne se situent pas là. L'un des rites les plus importants de la communauté kulung ne contient en effet pas de voyage. Les ancêtres des différents îlots de peuplement (tel) qui découpent l'espace habité sont en effet appelés sur une pierre tos, lieu des cultes agraires rendus par l'ensemble des propriétaires légitimes de la terre, les descendants des ancêtres défricheurs ${ }^{12}$. Car le territoire coïncide ici avec l'espace de production agraire hérité des ancêtres qui ont conquis la vallée sur la forêt, qui y sont morts et y furent enterrés, et dont la chair s'est fait terre. Leurs descendants en ont hérité des droits fonciers. Le territoire est nommé capkuwwa, le lieu des mânes, et ce sont ces ancêtres qui fournissent les récoltes faisant vivre les Kulung. Ce rite a pour objectif d'obtenir la prospérité et, pour effet, d'identifier et de redéfinir une communauté unie autour d'un territoire - entendu comme un espace considéré dans sa relation à une collectivité et comme le lieu d'exercice d'une autorité politique et foncière. Le rite tos débute dans la maison (autour du foyer et dans la cour) de l'officiant, qui personnifie la communauté, par une libation aux pierres du foyer ${ }^{13}$. Ensuite se met en place une petite procession qui connecte la maison de l'officiant à la pierre tos, où l'y rejoindront les maîtres ou maîtresses de maison descendants des ancêtres défricheurs. Lors de ce rite, il n'est donc pas de voyage: ce sont les ancêtres qui se déplacent des différents lieux du territoire qu'ils pourraient occuper pour venir sur la pierre. Comme la pierre du foyer, l'autel tos est un des rares autels fixes et permanents, contrastant ainsi avec les autels temporaires élaborés lors des voyages afin de ramener chez eux les esprits errants.

Les Kulung aiment à dire que, loin de chez soi, on tombe beaucoup plus facilement malade. Nous avons aussi vu que, lors de certains rituels, les âmes, 
passée une certaine distance, sont perdues, comme si le salut n'avait lieu que dans les frontières du territoire. Le message semble être: « reste chez toi si tu veux être bien portant ». Les seuls voyages positivement connotés sont ceux qui s'orientent vers le nord de la vallée (inscrits dans une géographie alors mal identifiée); mais même ceux-là ne sont pas sans danger: il faut se protéger des flèches décochées en certains lieux et faire attention à ne pas y laisser des âmes.

\section{Les pèlerinages}

Il existe pourtant un cas de voyage positivement connoté, et théoriquement sans danger. Il s'agit des déplacements effectués lors des « rassemblements 》 (melä), déplacement que l'on peut qualifier de pèlerinages, qui se tiennent périodiquement en des lieux particuliers, situés dans les hauteurs (cols, lacs, etc.), lieux d'une puissance auxquels certains fidèles se rendent à date précise pour leur rendre hommage, et espérer en tirer des bénéfices. Ces melā impliquent un déplacement, avec son lot d'incommodités, de souffrances, et l'idée qu'il faut payer de sa personne pour atteindre ce but. Par contraste avec les voyages rituels, le déplacement est réel, et bénéfique. Quoiqu'assez anciens, ces pèlerinages sont clairement des pratiques importées: tout le vocabulaire qui a trait à ces pratiques est népali (d'ailleurs, l'idée d'hommage à une puissance est absente des rites kulung) et leur déroulement est identique dans toute la région. Ce sont également des pratiques supra-ethniques (Chetri, Sherpas et autres groupes s'y rendent aussi).

Par contraste avec les rites tos mobilisant l'ancrage territorial de la communauté mono-ethnique sous l'auspice des ancêtres, ces pèlerinages - où la participation est volontaire - sont des carrefours pluri-ethniques, des lieux de rencontre et de confrontation fédérant des populations d'origines diverses autour d'un même objectif. Ces pèlerinages ont lieu en marge du territoire kulung, à ses frontières et au-delà. Ils sont pourtant créateurs d'un nouveau territoire : non plus celui de la communauté locale détentrice de la terre, mais celui de l'ensemble pluri-ethnique qui compose la nation népalaise, unie par le partage d'une même forme de ritualité contribuant à asseoir un territoire national. Effet communitas du pèlerinage, qui efface temporairement hiérarchie, statut et clivage pour faire émerger un sentiment de groupe uni dans son action pèlerine et sa dévotion (Turner 1975). Le pèlerinage est ici un mode unificateur à une échelle plus générale que les communautés locales, puisqu'il concourt à relier ces espaces disjoints. 


\section{Notes}

1. Sur cette histoire politico-foncière, voir CAPLAN 1970; PRADHAN 1991; FORBES 1996 ; EGL 2000.

2. Dans la littérature ethnologique sur l'Himalaya, on traduit ce type de déplacement verbal, présent parmi les groupes de langues tibéto-birmanes, par voyage rituel (ALIEN 1974; GAENSzIE 1994, 1999, 2001). Il évoque aussi le voyage chamanique (mais souvent plus abstrait, céleste ou infernal) et le voyage des psychopompes funéraires; nous y ferons référence.

3. Pour des raisons de place, cette description synthétique ne mentlonne que les éléments informatifs. Elle ne rend donc pas vraiment compte du rituel, quil contient nombre de répétitions, et quil est structuré par le rythme de la psalmodie, ce qui les rend très longues. J'ai pu observer, enregistrer et retranscrire la majeure partie des dix-sept rites et séquences rituelles comprenant des voyages, certains plusieurs fois entre 1995 et 2007. Parmi les marqueurs spécifiques de chaque rituel, notons le nom et qualificatif de l'esprit et/ou de la maladie qui motive le rite, une ligne mélodique et parfois une onomatopée répétée en boucle, les objets et la nourriture composant l'autel, la direction suivie et les étapes parcourues.

4. Tous ces rituels étant transmis et accomplis oralement, le discours incantatoire varie toujours, même si l'idéal kulung voudrait que les discours soient stables (d'où la surprise de mon assistant lors du travail de retranscription et d'analyse des rituels).

5. L'organisation de la madson chez les Kirant a fait l'objet de plusieurs travaux et est maintenant bien connue ; (SAGANT 1973, 1976; MACDOUGAL 1979 ; NICOLETTI 1995). Je me contenterai ici, en dehors de quelques informations nouvelles, de préciser ce qui est important pour notre propos.

6. Il s'agit là d'une simplification par soucl de clarté, car il faut en fait distinguer l'amont et l'aval de l'avant et l'arrière. La tradition voudrait que la porte centrale ouvre vers l'amont, du côté de la place des femmes. Mais cette observance faisalt que les hôtes avaient tendance à s'asseoir sur l'emplacement des femmes et être ainsi pollués par leurs menstrues, et de nos jours, la plupart des portes centrales ouvrent vers l'aval. Dans tous les cas, le déversoir et l'échelle qui montent à l'étage doivent se trouver vers l'amont. L'autel domestique dampe doit être orienté vers l'amont par rapport au foyer, pour pouvoir tourner dans le sens de l'amont lors des libations au foyer. Le critère avant/arrière ne détermine en fait que la place du foyer, de l'autel domestique et de l'auvent.

7. Le Nāgl est conçu comme un être (ou une fratrie de huit êtres) ophidien, résidant à Yowna, la source du monde, présent dès l'origine des temps, et qui fut allié aux Kulung à la suite du marlage de sa flle ou nièce avec Kokcilip, mariage à la suite duquel fut formée la civilisation. Appelé kul devata, divinitté lignagère, ce beau-père originel qui est, comme tout beau-père, potentiellement bénéflque et dangereux, reçoit un rite minimal une ou deux fois l'an, et un rite complexe et long une fois tous les trois ans.

8. Cette analyse de la verticalité chez les Rai a fait l'objet de plusieurs travaux: ALLEN 1972 ; GAENSZLE 1999 ; BICKEL 1999 et EBERT 1999. Notons par ailleurs que si chez les Kulung les formes verbales liées aux mouvements peuvent indiquer si le déplacement s'effectue vers le haut ou vers le bas, ce n'est pas le cas de celles utilisées dans les incantations des voyages rituels. 
9. Lors des funérailles, l'officiant mentionne: «Et voici le lieu de tes premiers ancêtres. Je te dis le discours des origines, je le fais, 8 mon enfant, que tes yeux regardent, que tes oreilles écoutent I Nous sommes ensemble à MinapongkoHalkumbu, ici. Ici, une eau puissante tombe avec force du ciel. Elle a formé le lac Diritti-Hotodi... ", puis narre tout le mythe d'origine.

10. Il existe deux autres rituels, réalisés en népali, lors desquels la rivière fait offlice d'égout et qui impliquent une dimension circulatoire concrète : le rite Hoplong dédié à l'esprit de la rivière Hongu qui traverse le territoire kulung, et celui dévolu aux esprits Sansari, « Celles du monde ». L'autel du premier est, à la fin du rite, jeté à l'eau pour que les infortunes qui y ont été déposées s'écoulent avec l'eau. Le second - adressé aux sept sœurs pour les uns, aux déesses courroucées ou aux vachères de Krishna pour d'autres, en tout cas aux porteuses de la peste et des autres épidémies - implique que les membres de chaque madsonnée déposent sur un palanquin qui passe de maison en maison des graines dans lesquelles on transfere les infortunes du corps et des maisons ; ce palanquin est emmené en un lieu d'où l'on voit le confluent de la rivière Shobolu afin de les y rejeter.

\section{Blbliographie}

ALIEN, N. (1972), « The Vertical Dimension in Thulung Classification ", Journal of the Anthropological Society of Oxford, III, pp. 81-94.

ALIEN, N. (1974), * The Ritual Journey : A Pattern Underlying Certain Nepalese Rituals $n$, in C. von Firer-Haimendorf, ed., Contributions to the Anthropology of Nepal, Warminster, Aris \& Phillips Ltd., pp. 6-22.
11. "Allons sur le chemin de toujours *, dit parfois l'officiant dans les incantations rituelles; on trouve aussi l'expression * le chemin des ancêtres, la parole des origines $*$, ou encore « je te renvoie comme/ avec le vent par le chemin des origines .

12. Les propriétaires légitimes de la terre sont les membres des clans localisés qui se sont vus reconnaitre un droit sur le kipat (régime foncier spécial) dans leur village. Les rites tos ne sont donc pas, à proprement parler, des rites villageois: 11 faut, pour y participer, être et du village et Kulung (ce quil exclut, de ce falt, les gens de castes parfois présents dans les villages kulung, et les Kulung récemment installés dans le village). Pour une description des rituels tos, voir SCHILMMER 2012.

13. L'autel tos peut être perçu comme un doublet collectif de la pierre lula qui se trouvait sur les terrasses (chumbolikher, lieu du mortier) de toutes les maisons pour battre le grain - renvoi à une combinaison entre le foyer et la terrasse. Avant de se rendre sur la pierre tos, l'officiant doit danser sur sa terrasse et dans sa cour, autour du mortier. Quant à l'offrande de patte de daim musqué et les libations de bière sur la pierre tos, elles rappellent directement les offrandes déposées sur le foyer et l'autel domestique.

BICKEL, B. (1999), * Cultural Formalism and Spatial Language in Belhara $\%$, in B. Bickel \& M. Gaenszle, eds., Himalayan Space: Cultural Horizons and Practices, Zürich, volkerkundemuseum der Universität Zürich, pp. 3-101.

CAPLAN, L. (1970), Land and Social Change in East of Nepal: A Study of HinduTribal Relations, Berkeley-Los Angeles, University of California Press. 
EBERT, K. (1999), « The Up-Down Dimension in Ral Grammar and Mythology ", in B. Bickel \& M. Gaenszle, eds., Himalayan Space: Cultural Horizons and Practices, Zürich, Völkerkundemuseum der Universität Zürich, pp. 105-131.

EGLI, W. (2000), « Below the Surface of Private Property: Individual Rights, Common Property, and the Nepalese kipas System in Historical Perspective ", European Bulletin of Himalayan Research, pp. 5-19.

FORBES, A.A. (1996), « The Discourse and Practice of Kipat », Kailash, 18 (1-2), pp. 39-80.

FORBES, A.A. (1998), « Sacred Geography on the Cultural Borders of Tibet ", in A.-M. Blondeau, ed., Tibetan Moutains Detties: Their Cults and Representation, Vienne, Verlag der Osterreichischen Akademie der. Wissenschaften, pp. 111-121.

GAENSZRE, M. (1994), "Journey to the Origin: A Root Metaphor in a Mewahang Rai Healing Ritual $n$, in N. Allen, ed., Anthopology of Nepal: Peoples, Problems and Processes, Kathmandou, Mandala Book Point, pp. 256-268.

GAENSZLE, M. (1999), «Travelling UpTravelling Down: The Vertical Dimension in Mewahang Rai Ritual Journeys ", in B. Bickel \& M. Gaenszle, eds., Hitmalayan Space: Cultural Horizons and Practices, Zitirich, Völkerkundemuseum der Universität Zïirich, pp. 135-163.

GAENSZLE, M. (2001), « Life Journeys : Rai Ritual Healers' Narratives on their Callings ", European Bulletin of Himalayan Research, pp. 20-21.

MACDOUGAL, Ch. (1979), The Kulunge Rai: A Study in Kinship and Marriage Exchange, Kathmandou, Ratna Pustak Bhandar.

MUMFORD, S.R. (1989), Himalayan Dialogue: Tibetan Lamas and Gurung Shamans in Nepal, Madison, University of Wisconsin Press.
NICOLETTI, M. (1995), «L'espace humain et l'espace non-humain. Contribution à l'étude de l'habitation et des conceptions quil y sont associées chez les Yakha Dewän du Népal oriental ", Studi e Materiali di Storia delle Religioni, LXI (n. s. XIX), pp. 151-202.

PRADHAN, K. (1991), The Gorkha Conquests: The Process and Consequence of the Untfication of Nepal with Particular Reference to Eastern Nepal, Calcutta, Oxford University Press.

SAGANT, P. (1973), « Prêtres limbu et catégorles domestiques , Kailash, I, n. 1, pp. 51-75.

SAGANT, P. (1981), « La tête haute. Maison, rituel et politique au Népal oriental ", in G. Toffin, ed., L'Homme et la Maison dans l'Himalaya, Paris, éditions du CNRS, pp. 149-175.

SCHLEMMER, G. (2004), « Vues d'esprits. La conception des esprits et ses implications chez les Kulung Rai du Népal », thèse de troisième cycle en ethnologie, Université Paris X-Nanterre.

SCHIEMMER, G. (2010a), « Rai, Khambu, Subba, Kirant, etc. : Ethnic Labels or Political and Land Tenure Categories? Logics of Identification of an Ensemble of Population in Nepal $n$, in C. Culas \& F. Robinne, eds., Interethnic Dymamics in Asta : Ethnonyms, Considering the Other though Ethnomyms, Territories and Rituals, Londres-New York, Routledge, pp. 42-56.

SCHLEMMER, G. (2010b), «The lllness is the Other People: Cross-Representations and Ritual Management of Alterity and Illness Among the Kulung (Nepal) ", in C. Culas, F. Robinne, eds., Interethnic Dynamics in Asia: Ethnonyms, Considering the Other Though Ethnonyms, Territories and Rituals, LondresNew York, Routledge, pp. 154-166.

SCHLEMMER, G. (2012), * Conquérants, fils du sol ou alliés de la forêt? Le rapport des Kulung au territoire et au 
pouvoir au prisme de leurs cultes au dieu du sol ", Moussons, 19, pp. 33-50. SIRCAR, D.C. (1960), Studies in the Geography of Ancient and Medieval India, Delhi, Motilal Banarsidass.
TURNER, V. (1975), «Pllgrimages as Social Processes ", in V. Turner, ed., Dramas, Fields, and Metaphors: Symbolic Action in Human Society, Ithica-Londres, Cornell University Press, pp. 167-230.

\section{Résumé}

Afin de révéler la manière dont les Kulung - une population de l'Himalaya népalais - découpent et conçoivent leur environnement géographique, organisent l'espace des lieux qu'lls retiennent et valorisent, l'article étudie des pratiques rituelles nommées natabom. Il s'agit de voyages immobiles, de parcours verbaux composés essentlellement de noms d'étapes en grande partie localisables, et dont la finalité est d'aller chercher des bienfaits ou de rejeter des infortunes. On présente les espaces parcourus, ce que les Kulung en disent et ce qu'ils y font, pour ensuite les comparer à d'autres pratiques rituelles (rites territoriaux et pelerinages). Il en ressort un rapport ambigu et complexe à l'extériorité et à l'altérité.

\section{Abstract}

\section{Immoblle Travelers}

The Mental Geography of the Kulung Rai (Nepal)

In order to reveal how Kulung (an Himalayan population living in Nepal) think their geographical environment, this article focuses on the study of ritual practices named natabom. These immobile travels, these verbal routes are mainly composed of a listing of names in large part localizable. The purpose of these travels is to seek benefits or reject misfortunes. The article present the places traveled, what Kulung say about them, and then it compare and contrast this natabom to others rituals practices (territorial cults and pilgrimages). It shows an ambiguous and complex relationship to exteriority and otherness. 